\title{
Influencia del flujo del líquido de diálisis en los parámetros de calidad de la hemodiafiltración en línea post-dilucional
}

\author{
Isabel Crehuet-Rodríguez, Marta Ramírez-Crehuet, Pilar Méndez-Briso-Montiano, Mª Teresa Mulero-San José
}

Servicio de Nefrología. Hospital Universitario Río Hortega. Valladolid. España

Como citar este artículo:

Crehuet-Rodríguez I, Ramírez-Crehuet M, Méndez-Briso-Montiano P, Mulero-San José, MT. Influencia del flujo del líquido de diálisis en los parámetros de calidad de la hemodiafiltración en línea post-dilucional. Enferm Nefrol. 2021 Ene-Mar;24(1):77-81

\section{Resumen}

Introducción: La hemodiafiltración en línea post-dilucional es una variante de hemodiálisis que, según diferentes estudios puede aumentar la supervivencia de los pacientes. Inicialmente, y a diferencia de la hemodiálisis convencional, se utilizaban flujos de dializado muy elevados (hasta $700-800 \mathrm{ml} / \mathrm{min}$ ); nuevos estudios cuestionaron la utilidad de elevar tanto este flujo en cuanto a eficacia depurativa.

Objetivo: Analizar las diferencias producidas en $\mathrm{Kt}$, $\mathrm{Kt} / \mathrm{V}$ y volumen de infusión en esta técnica utilizando flujo de baño de diálisis a $600 \mathrm{ml} / \mathrm{min}$ y a $500 \mathrm{ml} / \mathrm{min}$, así como evaluar la repercusión en el consumo de agua durante la sesión.

Método: Se incluyeron 22 pacientes crónicos dializados con hemodiafiltración en línea post-dilucional en monitores $\mathrm{Artis}^{\circledR}$. Se estudiaron 440 sesiones, 220 con cada flujo. Las variables estudiadas fueron edad, tiempo de permanencia en técnica, Kt, Kt/ $/ \mathrm{y}$ volumen de infusión.

Resultados: De los 22 pacientes 7 son mujeres, la mediana de edad es de 79,5 años (rango 40-86) y la media de permanencia: $25,13 \pm 10,51$ meses. Las medias con flujo de diálisis a $600 \mathrm{ml} / \mathrm{min}$ y a $500 \mathrm{ml} / \mathrm{min}$ fueron respectivamente: Kt:45,5 $\pm 9,4$ y 45,2 29,2 litros, Kt/V:1,52 $\pm 0,33$ y 1,51 $\pm 0,33$ y volumen de infusión: $21,84 \pm 4,50$ y $21,94 \pm 4,51$ litros.

\section{Correspondencia:}

Isabel Crehuet-Rodríguez

Email: crebel@hotmail.com
Conclusión: Según nuestros resultados se puede dializar en hemodiafiltración en línea post-dilucional con flujos de dializado de $500 \mathrm{ml} / \mathrm{min}$ sin menoscabo de los parámetros de calidad de hemodiálisis. La disminución del flujo del dializado de 600 a $500 \mathrm{ml} / \mathrm{min}$ en hemodiafiltración en línea post-dilucional no produce pérdida en los parámetros de calidad y permite de reducir el consumo de agua.

PALABRAS CLAVE: hemodiafiltración en línea; flujo líquido de diálisis; kt; volumen de infusión.

\section{Influence of the dialysate flow on the quality parameters of post-dilution line hemodiafiltration}

\begin{abstract}
Introduction: Online post-dilution hemodiafiltration is a variant of hemodialysis that, according to different studies, can increase patient survival. Initially, and unlike conventional hemodialysis, very high dialysate flows were used (up to $700-800 \mathrm{ml} / \mathrm{min}$ ), new studies questioned the usefulness of elevation to achieve purifying efficacy.

Objective: To analyze the differences produced in $\mathrm{Kt}, \mathrm{Kt} / \mathrm{V}$ and infusion volume in post-dilutional online hemodiafiltration using a dialysate flow at $600 \mathrm{ml} / \mathrm{min}$ and $500 \mathrm{ml} / \mathrm{min}$, as well as to evaluate the impact on the consumption of water during the session.
\end{abstract}


Method: 22 dialyzed chronic patients undergoing postdilutional hemodiafiltration on $\mathrm{Artis}^{\circledR}$ monitors were included. 440 sessions were studied, 220 with each flow. Age, time of permanence in technique, $\mathrm{Kt}, \mathrm{Kt} / \mathrm{V}$ and infusion volume were studied.

Results: Of the 22 patients, 7 were women, with a median age of 79.5 years (range 40-86) and a mean dialysis time of $25.13 \pm 10.51$ months. The mean values for dialysate flows of $600 \mathrm{ml} / \mathrm{min}$ and $500 \mathrm{ml} / \mathrm{min}$ were respectively: $\mathrm{Kt}: 45.5 \pm 9.4$ and $45.2 \pm 9.2$ liters, $\mathrm{Kt} / \mathrm{V}: 1.52 \pm 0.33$ and $1.51 \pm 0.33$, and infusion volume: $21.84 \pm 4.50$ and $21.94 \pm 4.51$ liters.

Conclusions: According to our results, on-line post-dilution hemodiafiltration can be dialyzed with dialysate flows of $500 \mathrm{ml} / \mathrm{min}$ without impairing the hemodialysis quality parameters. The decrease in dialysate flow from 600 to $500 \mathrm{ml} / \mathrm{min}$ in post-dilutional on-line hemodiafiltration does not produce a loss in quality parameters and allows to reduce water consumption.

KEYWORDS: on-line hemodiafiltration; dialysate flow; $\mathrm{Kt}$; infusion volume.

\section{Introducción}

La hemodiafiltración en línea post-dilucional (HDFOL-pd) hasta el momento y avalada por diversos estudios, resulta ser la técnica de hemodiálisis (HD) más eficaz. Comparada con otros tipos de HD, es la que mejores resultados consigue en depuración de todo tipo de moléculas, amiloidosis asociada a la diálisis, tolerancia hemodinámica, hiperfosforemia, desnutrición, etc. por lo que debe ser la primera opción de hemodiálisis ${ }^{1}$.

A diferencia de la HD convencional, para formar el dializado utiliza agua ultrapura, prácticamente estéril, cuya contaminación máxima por bacterias es $\leq 0,1$ $\mathrm{UFC} / \mathrm{ml}$ y por endotoxinas $\leq 0,03 \mathrm{UE} / \mathrm{ml}$. Actualmente para todas las modalidades de $\mathrm{HD}$ se recomienda la utilización de líquido ultrapuro, porque el uso habitual del mismo es la mejor forma de prevenir o retrasar ciertas complicaciones relacionadas con dichas técnicas a largo plazo ${ }^{2}$.

Recientemente están apareciendo nuevos estudios basados en técnicas mixtas, que parecen mostrar también buenos resultados ${ }^{3}$.

Uno de los factores que condicionan la morbi-mortalidad en estos pacientes es la dosis de diálisis. Pero, ¿cuál es la dosis adecuada de diálisis? La medición ha ido variando a lo largo del tiempo. En la actualidad, tras el Estudio Multicéntrico Americano y consensuado en las guías de nefrología tanto americanas (K-DOQI) ${ }^{4}$ como europeas ${ }^{5}$ y nacionales ${ }^{6}$, es aquella que logra un $K t / V \geq 1,3$ y un porcentaje de reducción de urea del 70\%. En 1999, Lowrie y colaboradores, proponen utilizar el Kt para medir dosis de diálisis y mortalidad y hablan de conseguir un Kt de 40-45 litros (L) para mujeres y 45-50 L para hombres ${ }^{7}$. Las ventajas de utilizar el Kt son la posibilidad de medirlo en tiempo real en cada sesión, es específico para medir la dosis de diálisis, es independiente del volumen de distribución de urea, con lo cual no se ve afectado por el estado nutricional del paciente, lo cual es importante ya que un gran número de pacientes sufre desnutrición ${ }^{8}$.

En las técnicas convectivas, otro parámetro importante que influye en la dosis de diálisis es el volumen de infusión (VI), que junto a la sobrecarga hídrica interdiálisis (ultrafiltración), conforman el volumen convectivo total (VCT) de la sesión. La cantidad de volumen administrado parece ser decisiva en la mejora de la supervivencia. Varios estudios multicéntricos, con una gran muestra de pacientes, demostraron una reducción de la mortalidad con las técnicas de HDFOL-pd respecto a las técnicas de HD, cuando el VCT era muy elevado.

Los primeros resultados de HDFOL fueron publicados por Canaud en $1987^{\circ}$. Posteriormente fueron apareciendo varios estudios que demostraban la eficacia de la HDFOL respecto a la HD. Así el estudio ESHOL (High-Efficiency Postdilution Online Hemodiafiltration Reduces All-Cause Mortality in Hemodialysis Patients), con una muestra de 906 pacientes durante 3 años, observaron que en los que se aplicaba HDFOL-pd, la mediana del VI fue de 20-22 litros y el VCT fue de 23-24 litros ${ }^{10}$. En el análisis primario se demostró que en el grupo de HDFOL-pd se redujo un 30\% la mortalidad global, un 33\% la cardiovascular y un 55\% la de causa infecciosa. También observaron una mayor estabilidad hemodinámica durante la sesión y menor tasa de hospitalización. En un análisis secundario la mortalidad global se redujo hasta en un $40 \%$ cuando los pacientes recibieron un VCT entre 23-25 litros y en un $45 \%$ si recibieron más de 25 litros. En el otro grupo de pacientes se realizaron HD de alto flujo. De los análisis secundarios de estos estudios se puede extraer la recomendación de utilizar la HDFOL-pd y obtener un VI >20-22 litros.

Por lo tanto, se puede decir que para obtener una "dosis adecuada de diálisis" necesitamos obtener un Kt de 40$45 \mathrm{~L}$ en mujeres y $45-50 \mathrm{~L}$ en varones; $\mathrm{Kt} / \mathrm{V} \geq 1,3$ y un VI $>20-22$ L. 
Ahora bien, para conseguir estos valores además de la técnica hay que tener en cuenta otros parámetros como el flujo de sangre $\left(Q_{b}\right)$, que en técnicas convectivas debe de ser elevado, a ser posible por encima de $350 \mathrm{ml} / \mathrm{min}$, flujo del dializado $\left(Q_{\mathrm{d}}\right)$, que en sus inicios era de $700-800 \mathrm{ml} / \mathrm{min}$, bastante superior a los $500 \mathrm{ml} /$ min que se emplean en la HD convencional, tiempo de la sesión, dializador, etc.

Es evidente que, cualquier técnica de hemodiálisis requiere un gran consumo de agua para formar el líquido de diálisis (LD); para una sesión de 4 horas, a un $Q_{d}$ de $500 \mathrm{ml} / \mathrm{min}$, necesitaremos como mínimo $120 \mathrm{~L}$ de agua, contando única y exclusivamente la sesión, sin tener en cuenta el agua necesaria para la preparación del baño ni para la desinfección y lavado del monitor, que se necesitaría independientemente de la técnica. Si se utilizan Qd más altos, por ejemplo $600 \mathrm{ml} / \mathrm{min}$ en HDFOL, se necesitan 144 L/sesión.

Dado el importante volumen de agua utilizado para estas técnicas, en nuestra Unidad, cuya pauta para HDFOL-pd en los monitores Artis $^{\circledR}$ es mantener un $Q_{d}$ de $600 \mathrm{ml} / \mathrm{min}$, nos planteamos rebajarlo a $500 \mathrm{ml} / \mathrm{min}$ y realizar un estudio cuyo objetivo principal es analizar las diferencias producidas en los parámetros de calidad de diálisis (Kt, Kt/V y VI) en HDFOL-pd con ambos flujos y como objetivo secundario evaluar el impacto en el consumo de agua.

\section{Material y Método}

Estudio cuasi experimental de medida antes y después, realizado en la Unidad de Diálisis del Hospital Universitario Río Hortega de Valladolid. La recogida de datos se realizó a lo largo del primer semestre del año 2017. Se incluyeron 22 pacientes en programa de hemodiálisis crónica que se dializaban, al menos desde hacía un mes, con técnica HDFOL-pd en monitores Artis ${ }^{\circledR}$, mayores de edad y que aceptaron participar en el estudio de forma voluntaria. Se analizaron solamente los datos recogidos en las sesiones en las que no hubo ninguna incidencia, un total de 440 sesiones: 220 (10 sesiones/ paciente) se realizaron con un Qd de $600 \mathrm{ml} / \mathrm{min}$ y las otras 220 ( 10 sesiones/paciente) con $Q_{d}$ de $500 \mathrm{ml} / \mathrm{min}$.

Para la recogida de datos se elaboró una hoja diseñada específicamente para el estudio, en la que se recogieron variables sociodemográficas (sexo y edad), tiempo de permanencia en HDFOL-pd, parámetros como el flujo de sangre, tiempo de la sesión, acceso vascular, diali- zador, calibre de agujas de punción de FAV, Kt, Kt/V, VI y monitores de hemodiálisis.

Inicialmente se recogieron los datos de todas las sesiones correspondientes a un mes (alrededor de 290 sesiones), para poder determinar el $Q_{b}$ estable más alto posible y que nos proporcionara el menor número de interferencias durante la sesión; era muy importante mantenerlo constante, pues era uno de los que más podían sesgar el estudio. El resto de parámetros que queríamos mantener constantes (tiempo de la sesión, dializador, agujas, monitor, etc.) eran más fácilmente controlables. Una vez determinado este $Q_{b}$ en cada paciente, comenzamos la recogida de datos, manteniendo constantes los siguientes parámetros (Tabla 1): Flujo de sangre, tiempo de la sesión, dializadores (de alta permeabilidad y purgados de forma automática con una ultrafiltración en el cebado de 4 litros para todos ellos, no pudiendo intervenir el personal), accesos vasculares, calibre de las agujas y monitores.

Las mediciones del Kt se hacían por dialisancia iónica a lo largo de la sesión, el Kt/V se obtenía introduciendo el volumen de distribución de urea (V) de forma manual. La medición de $\mathrm{V}$ se realizó mediante bioimpedancia eléc-

Tabla 1. Parámetros basales del estudio

\section{Parámetros / ( ${ }^{0}$ Pacientes)}

\begin{tabular}{|c|c|}
\hline FLUJO DE SANGRE & TIEMPO SESIÓN \\
\hline - $330 \mathrm{ml} / \mathrm{min} /$ (7) & - 3:00 horas / (1) \\
\hline - $350 \mathrm{ml} / \mathrm{min} /(7)$ & - 3:30 horas / (10) \\
\hline - $380 \mathrm{ml} / \mathrm{min} /$ (2) & - 4:00 horas / (8) \\
\hline - $400 \mathrm{ml} / \mathrm{min} /(6)$ & - 4:30 horas / (3) \\
\hline $\begin{array}{l}\text { DIALIZADOR } \\
\text { - Xevonta }{ }^{\circledR} /(4) \\
\text { - Revaclear } 400^{\circledR} /(3) \\
\text { - Evodial } 2.2^{\circledast} /(8) \\
\text { - Polyflux } 210 \mathrm{H}^{\circledR} /(3) \\
\text { - Fx } 80^{\circledR} /(4)\end{array}$ & $\begin{array}{l}\text { ACCESO VASCULAR } \\
\text { - FAV: nativas } \\
\text { - radio-cefálica / (6) } \\
\text { - húmero cefálica / (3) } \\
\text { - húmero-basílica / (2) } \\
\text { - CVCT: palindrómicos } \\
\text { - vena yugular derecha / (6) } \\
\text { - vena yugular izquierda / (4) } \\
\text { - vena femoral derecha / (1) }\end{array}$ \\
\hline $\begin{array}{l}\text { CALIBRE DE AGUJAS } \\
\text { - } 15 \mathrm{G}(2 \mathrm{~mm} \emptyset) /(8) \\
\text { - } 14 \mathrm{G}(1,8 \mathrm{~mm} \emptyset) /(3)\end{array}$ & $\begin{array}{l}\text { MONITORES ARTIS } \\
\text { - El mismo en cada paciente } \\
\text { durante todo el estudio }\end{array}$ \\
\hline
\end{tabular}


trica con el $\mathrm{BCM}^{\circledast}$ de Fresenius Medical Care y el VI lo medía el monitor en tiempo real. Los datos se recogían al finalizar cada sesión.

Para el análisis estadístico se utilizó el programa SPSS 15.0 para Windows. Las variables cuantitativas se expresaron como media \pm desviación estándar o mediana y rango. Las diferencias de medias se analizaron mediante la $t$ de Student para muestras apareadas y la correlación entre variables con la $r$ de Pearson. Se consideró como significación estadística un valor $p<0,05$.

La investigación fue aprobada por el Comité de Ética de Investigación Clínica del Hospital Universitario Río Hortega de Valladolid, con el código CEIC:24/17. El estudio se llevó a cabo cumpliendo la Ley orgánica 3/2018, de 5 de diciembre de protección de datos personales y garantía de los derechos digitales.

\section{Resultados}

Se analizaron un total de 220 sesiones de $H D$ con un $Q_{d}$ de $600 \mathrm{ml} / \mathrm{min}$ y otras 220 con un Qd de $500 \mathrm{ml} / \mathrm{min}$, en una muestra de 22 pacientes de los que 7 eran mujeres.

La mediana de la edad fue de 79,5 (rango 40-86) años.

La media del tiempo en HDFOL-pd fue de $25,13 \pm 10,51$ meses.

Las medias del $\mathrm{Kt}$, con $\mathrm{Q}_{\mathrm{d}}$ a $600 \mathrm{ml} / \mathrm{min}$ y a $500 \mathrm{ml} / \mathrm{min}$ fueron respectivamente de: 45,5 $\pm 9,4$ y 45,2 $\pm 9,2 \mathrm{~L},(p=$ 0,004 ) alcanzándose la significación estadística, aunque estas diferencias no eran clínicamente relevantes.

Las medias del $\mathrm{Kt} / \mathrm{V}$ con $\mathrm{Q}_{\mathrm{d}}$ a $600 \mathrm{ml} / \mathrm{min}$ y a $500 \mathrm{ml} /$ min fueron de $1,52 \pm 0,33$ y $1,51 \pm 0,33$ respectivamente $(p=0,091)$.

En cuanto al VI, se alcanzó un valor medio de $21,84 \pm 4,50$ $L$ a un $Q_{d}$ de $600 \mathrm{ml} / \mathrm{min}$ y de $21,94 \pm 4,51 \mathrm{~L}$ con un $Q_{d}$ de $500 \mathrm{ml} / \mathrm{min}(\mathrm{p}=0,195)$.

\section{Discusión}

Se recogieron datos de cerca de 750 sesiones de HDFOL-pd, de las cuales se descartaron alrededor de 300 por problemas tales como: averías en el monitor, indisposición del paciente durante la sesión por hipotensión, calambres, etc., que obligaron a reducir el tiempo y, por lo tanto, no se utilizaron los datos de esas sesiones. En otras ocasiones, se produjo una disfunción del acceso que necesitó la inversión de las líneas, y aunque todos los catéteres utilizados eran de tipo palíndrómico, en los cuales la recirculación es prácticamente igual con las ramas en posición normal o en posición invertida, tal como se había demostrado en un estudio previo ${ }^{11}$, también se descartaron los datos de estas sesiones para evitar sesgos.

A diferencia de otras publicaciones, en las que se consiguen $Q_{b}$ superiores a $400 \mathrm{ml} / \mathrm{min}^{12}$, en nuestro caso no obtuvimos $Q_{b}$ tan elevados (media de $Q_{b}: 360 \pm 28,78$ $\mathrm{ml} / \mathrm{min}$ ). Hay que tener en cuenta que los pacientes de nuestro estudio tenían una edad bastante más avanzada y, además, la mitad de ellos portaban catéteres venosos centrales como acceso vascular, en los que es más difícil conseguir $Q_{b}$ tan altos. Respecto al tiempo de la sesión, algunos autores manifiestan que si se disminuye el $Q_{d}$ habría que aumentar el tiempo aunque solo fueran unos pocos minutos ${ }^{13}$; en nuestro caso, la media de duración de las sesiones era de $227,72 \pm 23,89 \mathrm{~min}$, inferior a los estudios mencionados en los que se alcanzan tiempos de sesión superiores a 245 minutos. No obstante, logramos dosis adecuadas de diálisis con ambos flujos, y aunque la diferencia producida en el Kt sí fue estadísticamente significativa, ésta no resultó clínicamente relevante, dado que se obtuvieron en ambos casos valores de Kt dentro de los rangos establecidos. Desconocemos los factores a los que atribuir estas diferencias, pudiendo ser éstos objeto de próximos estudios.

Un aspecto a tener en cuenta es el impacto que puede suponer la modificación del flujo del baño de diálisis en el consumo de agua durante la sesión. Un paciente con un tratamiento de HDFO L-pd de 4 horas de duración, 3 días a la semana, a un $Q_{d}$ de $600 \mathrm{ml} / \mathrm{min}$ consume, aproximadamente, unos $22.500 \mathrm{~L}$ de agua ultrapura al año (144 litros/sesión x 3 × 52 semanas). Si lo disminuimos a $500 \mathrm{ml} / \mathrm{min}$ el gasto de agua ultrapura sería de unos 18.700 L ( 120 L/sesión x 3 x 52 semanas), con lo cual el ahorro de agua ultrapura que se puede conseguir es de unos 3800 - 4000 L/año/paciente. Si, como concluyen algunos estudios, la cantidad de agua potable que se necesita para obtener $1 \mathrm{~L}$ de agua ultrapura es de $2 \mathrm{~L}^{20}$, el ahorro de agua potable sería enorme, aproximadamente unos 7.600-8000 L/paciente/año.

Posiblemente estos resultados se podrían extrapolar a otros monitores que utilizan $Q_{d}$ más elevados. En nuestra Unidad de HD, con una prevalencia de 50 pacientes/ año, al pasar de un $Q_{d}$ de $600 \mathrm{ml} / \mathrm{min}$ a $500 \mathrm{ml} / \mathrm{min}$, el ahorro anual sería de unos $187.000 \mathrm{~L}$ de agua ultrapu- 
ra $(50 \times 24 \times 156=187.200 \mathrm{~L}$, donde 50 es el número de pacientes, 24 es el número de litros de agua ultrapura que se ahorran por sesión y 156 las sesiones que normalmente se realiza un paciente al año) y según los estudios ya mencionados, el doble de agua potable, alrededor de $375.000 \mathrm{~L}$.

La principal limitación de nuestro estudio reside en el tamaño muestral, que por ser pequeño impide corroborar la hipótesis planteada con la potencia suficiente.

A partir de los resultados obtenidos podemos concluir que con la disminución del flujo del dializado de 600 a $500 \mathrm{ml} / \mathrm{min}$ en hemodiafiltración en línea post-dilucional se puede obtener una dosis de diálisis adecuada, lo que permite optimizar dichos flujos con el fin de reducir el consumo de agua.

Recepción: 18-01-21

Aceptación: 3-02-21

Publicación: 30-03-21

\section{Bibliografía}

1. Maduell F. Hemodiafiltración en linea. En: Lorenzo V, López Gómez JM. Nefrología al día. Hemodiafiltración en línea. [consultado 04 Mar 2020]. Disponible en: https://www.nefrologiaaldia.org/271.

2. Pérez-García R, García Maset $R$, González Parra E, Solozábal Campos C, Ramírez Chamond R, Martín-Rabadán $\mathrm{P}$, et al; Comisión de Expertos de la Sociedad Española de Nefrología para la creación de la Segunda Edición de la Guía de Gestión de Calidad del Líquido de Diálisis. Nefrologia. 2016;36(3):el-e52.

3. De Sequera P, Albalate M, Pérez-García R, Corchete E, Puerta M, Ortega Díaz M, Alcázar R et al. Comparación de la eficacia de dos modalidades de hemodiafiltración en línea: mixta frente a posdilucional. Nefrología 2013;33(6):779-87.
4. National Kidney Foundation. KDOQI Clinical Practice Guideline for Hemodialysis Adequacy: 2015 update. Am J Kidney Dis. 2015;66(5):884-930.

5. European Renal Association-European Dialysis and Transplant Association. European Best Practice Guidelines for Hemodialysis. Nephrol Dial transplant 2002; 17(Suppl 7):S17-21.

6. Maduell F, García M, Alcázar R. Dosificación y adecuación del tratamiento dialítico. Guías SEN. Guías de centros de Hemodiálisis. Nefrología. 2006;26 (Supl 8):S15-21.

7. Lowrie EG, Cherton GN, Lew NL, Lazarus JM, Owen $W F$. The urea (clearance $x$ dialysis time) product (Kt) as an outcome-based measure of hemodialysis dose. Kidney Int 1999;56:729-37.

8. Molina Núñez $M$, Roca Meroño $S$, Alarcón Jiménez RM, García Hernández MA, Jimeno Griño C, Álvarez Fernández GM et al. Cálculo del Kt como indicador de calidad en el área de adecuación en hemodiálisis. Nefrología 2010;30(3):331-6.

9. Canaud B, Nguyen QV, Argiles A, Polito C, Polascheoo HD, Mion C. Hemodiafiltration using dialysate substituction fluid. Artif organs 1987;11(2):188-90.

10. Maduell F, Moreso F, Pons M, Ramos R, Mora-Macià J, Carreras J et al; ESHOL Study Group. High-efficiency postdilution online hemodiafiltration reduces all-cause mortality in hemodialysis patients. J Am Soc Nephrol. 2013;24(3):487-97.

11. Crehuet Rodríguez I, Méndez Briso-Montiano P, Mulero San José T, Bernárdez Lemus $M$, Jiménez Maldonado A, Toribio Manrique B. Recirculación de la sangre durante la sesión de hemodiálisis en el catéter tunelizado Palindrome TM. Enferm Nefrol 2012;15(1):22-7.

12. Maduell $F$, Ojeda $R$, Arias-Guillén $M$, Fontseré $N$, Vera M, Massó $E$ et al. Optimización del flujo de diálisis en la hemodiafiltración on-line. Nefrología. 2015;35(5):473-8.

13. Albalate $M$, Sequera $P$, Pérez-García $R$, Corchete $E$, Alcázar R, Ortega M, Puerta M. ¿Cuál es el flujo de baño óptimo en la hemodiafiltración on-line posdilucional? Nefrología. 2015:35(6):533-8.

Este artículo se distribuye bajo una Licencia Creative Commons Atribución-NoComercial 4.0 Internacional. https://creativecommons.org/licenses/by-nc/4.0/

Open Access (c) () \& 